\title{
EDITORIAL
}

\section{El vaso medio lleno}

\author{
Juan Pablo Cruz Quiroga ${ }^{\star}$
}

1. Radiólogo. Pontificia Universidad Católica de Chile. Santiago, Chile.

Los múltiples cambios que esta pandemia ha generado en los distintos quehaceres de nuestro entorno social, laboral y familiar han sido tema de innumerables columnas, editoriales y artículos de las más variadas índoles. Se hace complejo buscar temas de impacto para comentar en una editorial que no tengan relación con las nuevas maneras en que nos hemos visto obligados a convivir.

Es por eso que vuelvo a escribir sobre el tema nuevamente, con ya varias semanas de aislamiento o cuarentena en distintos grados, tratando de exponer los elementos positivos y las futuras oportunidades que seguramente vendrán a medida que las restricciones vayan desapareciendo.

Del punto de vista laboral y de presencia de nuestra especialidad, la implementación casi obligatoria de buenos sistemas de teletrabajo ha facilitado la noción del radiólogo disponible, contando en casa con casi las mismas herramientas que en las estaciones de trabajo de los lugares físicos de trabajo. Con eso, se hace más factible la flexibilidad horaria y la capacidad de entregar respuestas rápidas y certeras, sin los riesgos de visores web con limitaciones intrínsecas que pueden limitar el rendimiento diagnóstico. Ha hecho que podamos estar en el proceso sin necesariamente estar presentes físicamente.

Del punto de vista docente, los sistemas para impartir clases remotas (principalmente vía Zoom o GoToMeeting), nos han permitido ampliar la audiencia, siendo posible que los residentes y alumnos puedan participar de la clase desde cualquier plataforma y desde cualquier lugar, eliminando los problemas logísticos de la necesidad de auditorios adecuados, disponibilidad horaria de estos y traslado de los participantes (en especial de los programas que ocurren en múltiples centros), permitiendo así que los espacios asignados no se alarguen, disminuyendo los tiempos muertos.

También la Sociedad Chilena de Radiología ha podio impartir cursos en línea y webinars con una gran asistencia y participación. Facilita la participación de conferencistas, pudiendo tener acceso a académicos internacionales sin incurrir en los costos monetarios y de tiempo que significa cada invitado, además de ampliar la participación de radiólogos de regiones que ya no dependen de tener que viajar a la capital, pudiendo descentralizar eventualmente nuestra especialidad. Como mencionaba en la editorial del primer número de la revista, abre la posibilidad de un currículum nacional común a las residencias nacionales. Todas estas actividades y la facilidad de participar en ellas fomentan el sentido de pertenencia de los radiólogos como grupo, y la SOCHRADI es el lugar natural donde podemos confluir. Queda el gran desafío del año aún para nuestra Sociedad, que es el Congreso virtual. El número de asistentes a nuestro congreso ha ido en constante alza, y tenemos la oportunidad única este año de que todos los interesados puedan "estar" y participar.

Dentro de lo que considero que son buenas noticias para los que estamos interesados y participamos en la docencia y algo de academia, existen si algunas banderas rojas, por lo que quisiera terminar con un par de recomendaciones. La flexibilidad horaria requiere un orden y calendarización, cosa que nuestro trabajo no interfiera con aquellos espacios del día que antes teníamos asignados a la familia o al ocio. La separación dentro de la casa de espacios laborales y no laborales es también muy importante para poder finalmente decidir terminar de trabajar en el día a día. La sobre oferta de webinars y cursos en línea también puede generar una sensación de ansiedad y de estar "perdiendo oportunidades" por no poder atender, por lo que se hace necesario elegir aquellos en que se esté seguro de poder ir y aquellos de mayor interés o impacto.

Finalmente, los cambios de circunstancias nos obligan a aprender y a adaptarnos, y somos parte de una especialidad que tiene todas las capacidades para poder seguir contribuyendo, enseñando y creciendo, pese a todas las limitaciones actuales.

Cruz JP. El vaso medio lleno. Rev Chil Radiol 2020; 26(3): 85.

*Correo electrónico: Juan Pablo Cruz Q / jpcruz@med.puc.cl 